\title{
STUDIES ON THE PHYSIOLOGY OF HUNGER. I. THE EFFECT OF INTRAVENOUS ADMINISTRATION OF GLUCOSE ON GASTRIC HUNGER CONTRACTIONS IN MAN ${ }^{1}$
}

\author{
By ALBERT J. STUNKARD AND HAROLD G. WOLFF WITH THE TECHNICAL ASSISTANCE \\ of ALBA PLESCIA
}

\author{
(From the Study Program in Human Health and the Ecology of Man, Departments of Medicine \\ and Psychiatry, New York Hospital-Cornell Medical Center, New York, N. Y.)
}

(Submitted for publication December 27, 1955; accepted May 17, 1956)

The introduction of measures for the recording of gastric hunger contractions half a century ago laid the foundations for the modern study of appetite and hunger $(1,2)$. The finding that intravenous administration of glucose could abolish these contractions in fasting dogs led Bulatao and Carlson to postulate that "(lack of) availability of carbohydrates for utilization . . . is an important factor in the genesis of the gastric hunger contractions" (3). Further evidence for this hypothesis was not forthcoming, and later reports of the effects of glucose administration on gastric motility have been inconsistent, and even in conflict (4-6).

Recently this problem has been reopened by Mayer's proposal of a "glucostatic" theory of the regulation of food intake which ascribes hunger to a depletion of the carbohydrate stores of the body, and satiety to the replenishment of these stores (7, 8). According to this theory the status of the carbohydrate stores is signalled to the hypothalamic food regulatory centers by the rate at which glucose is utilized.

Since experiments of the type performed by Bulatao and Carlson should provide a test of the glucostatic theory, it seemed important to repeat these studies in man to determine with greater assurance the effects of glucose on gastric hunger contractions. The peripheral removal of glucose provides an index of the metabolic events accompanying such injection. Accordingly, peripheral capillary-venous glucose differences were determined at intervals throughout the experiment, and these values were correlated with the effects of glucose injection on gastric hunger contractions.

1 This work was supported in part by the Research and Development Division, Office of the Surgeon General, Department of the Army, Contract No. DA-49-007-MD524.

\section{METHODS AND MATERIALS}

Twenty-three adult persons served as subjects for this investigation, 9 on more than one occasion. Three were healthy volunteers, 20 were patients from the general medical clinic of the New York Hospital, 5 with a diagnosis of diabetes mellitus, 15 with diagnoses as listed in Tables I and II.

Each subject was studied in the morning after an overnight fast. At the beginning of the experiment a Levine tube with attached gastric balloon was inserted into the stomach. The balloon was inflated to a pressure of 10 $\mathrm{cm}$. of water and the tube was withdrawn until resistance was encountered at the cardia. The tube was then taped into place at the nose. Gastric contractions were recorded on a kymograph by means of a water manometer. An intravenous infusion of isotonic sodium chloride was begun and base-line capillary and venous blood samples were drawn. Carefully standardized control periods of from three to five hours allowed assessment of the subject's pattern of gastric activity as well as the effect on gastric motility of a rapid (3-minute) injection of $70 \mathrm{ml}$. of saline through the infusion tubing. In only one subject was such saline injection followed by inhibition of gastric contractions; he was excluded from the study.

During a period of vigorous gastric contractions, 50 $\mathrm{ml}$. of a 50 per cent solution of glucose were administered through the infusion tubing over a period of 3 minutes. In half the cases this was associated with transient discomfort in the area above the needle; the remaining subjects were either unaware of the injection or experienced no discomfort. There was no correlation between such reactions and subsequent inhibition of gastric hunger contractions.

Venous and capillary blood samples were obtained according to two different schedules; at 10,30 and 50 minutes, and at 20,40 and 60 minutes following completion of the glucose injection. To conserve space all of these values are not recorded in the tables. Venous blood was drawn with minimal stasis from an antecubital vein, and capillary blood was obtained almost simultaneously by direct pipetting from a finger tip of the same extremity after cutaneous puncture. Capillary blood has been shown to have the same glucose content as arterial blood (9-11).

Glucose in whole blood was determined by the method of Somogyi as modified by Nelson (12-14). Determinations were done in duplicate on $0.2-\mathrm{ml}$. samples. Stand- 


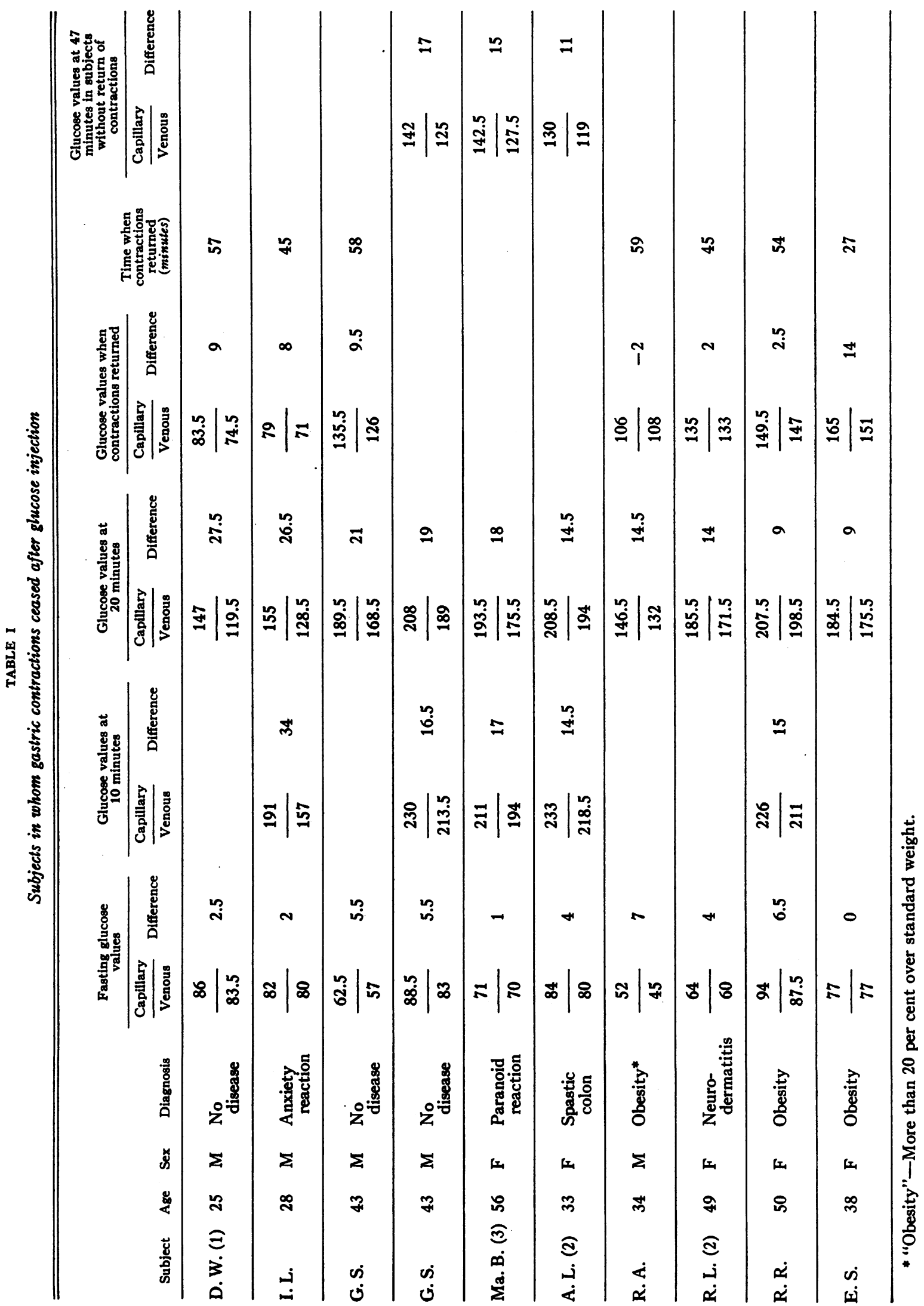




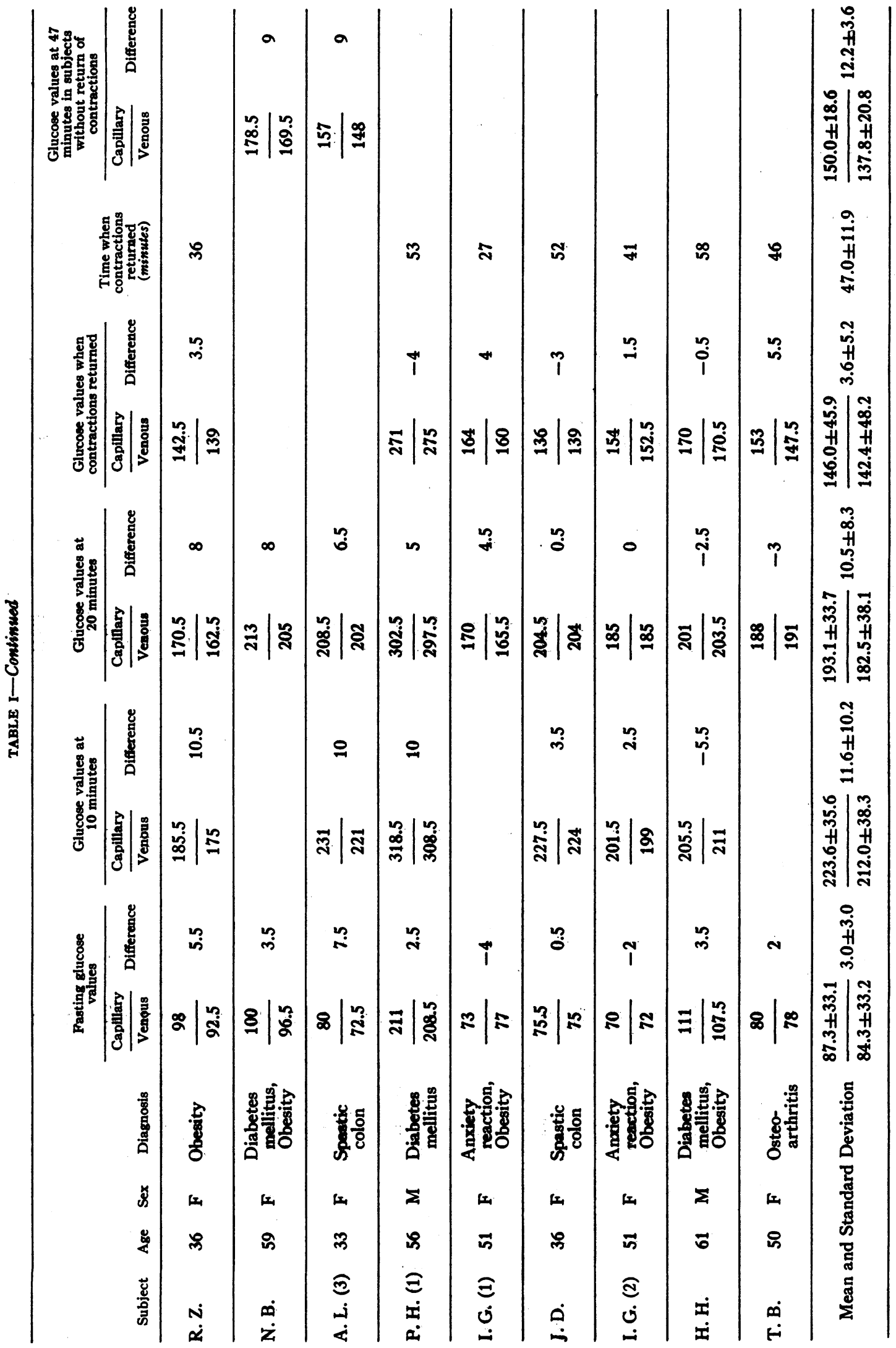


TABLE II

Subjects in whom gastric contractions persisted after glucose injection

\begin{tabular}{|c|c|c|c|c|c|c|c|c|c|}
\hline \multirow[b]{3}{*}{ Subject } & \multirow[b]{3}{*}{ Age } & \multirow[b]{3}{*}{ Sex } & \multirow[b]{3}{*}{ Diagnosis } & \multicolumn{2}{|c|}{$\begin{array}{c}\text { Fasting glucose } \\
\text { values }\end{array}$} & \multicolumn{2}{|c|}{$\begin{array}{c}\text { Glucose values at } \\
10 \text { minutes }\end{array}$} & \multicolumn{2}{|c|}{$\begin{array}{l}\text { Glucose values at } \\
20 \text { minutes }\end{array}$} \\
\hline & & & & Capillary & & Capillary & & Capillary & \\
\hline & & & & Venous & Difference & Venous & Difference & Venous & Difference \\
\hline \multirow{2}{*}{ A. L. (1) } & \multirow{2}{*}{33} & \multirow{2}{*}{$\mathbf{F}$} & \multirow{2}{*}{$\begin{array}{l}\text { Spastic } \\
\text { colon }\end{array}$} & 79 & \multirow{2}{*}{5} & & & 204.5 & \multirow{2}{*}{20} \\
\hline & & & & 74 & & & & 184.5 & \\
\hline \multirow{2}{*}{ L. T. } & \multirow{2}{*}{45} & \multirow{2}{*}{$\mathbf{M}$} & \multirow{2}{*}{$\begin{array}{c}\text { (Treated) } \\
\text { Anemia }\end{array}$} & 88 & \multirow{2}{*}{3.5} & 191.5 & \multirow{2}{*}{13} & 179.5 & \multirow{2}{*}{11.5} \\
\hline & & & & 84.5 & & 178.5 & & 168 & \\
\hline \multirow{2}{*}{ P. H. (2) } & \multirow{2}{*}{56} & \multirow{2}{*}{$\mathbf{M}$} & \multirow{2}{*}{$\begin{array}{c}\text { Diabetes } \\
\text { mellitus }\end{array}$} & 111 & \multirow{2}{*}{0} & & & 177 & \multirow{2}{*}{11} \\
\hline & & & & 111 & & & & 166 & \\
\hline D w (2) & 25 & $\mathbf{M}$ & No dicence & 85 & 3 & 178.5 & 75 & 165.5 & 105 \\
\hline 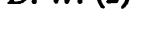 & 20 & 20 & 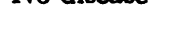 & 82 & & 171 & 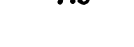 & 155 & 10.0 \\
\hline$M G$ & 25 & $\mathbf{M}$ & No dicence & 67.5 & -1 & & & 166.5 & 65 \\
\hline 10.0 & 20 & 20 & 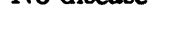 & 68.5 & & & & 160 & 0.0 \\
\hline $\mathrm{P} H$ (3) & 56 & $\mathbf{M}$ & & 77 & 10 & & & 148.5 & 6 \\
\hline 2.0078 & 0 & No & mellitus & 67 & 20 & & & 142.5 & 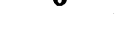 \\
\hline M B (1) & 26 & $\mathbf{F}$ & & 186 & 10 & & & 259 & 35 \\
\hline (2) & 20 & 2 & mellitus & 176 & 20 & & & 255.5 & 0.0 \\
\hline $\mathrm{V}, \mathbf{N}$ & 50 & $\mathbf{F}$ & & 94 & 15 & & & 213.5 & \\
\hline 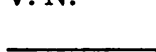 & 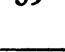 & F & arthritis & 92.5 & 1.0 & & & 210 & 0.0 \\
\hline$M a \mathbf{M}$ (2) & 56 & $\mathbf{F}$ & & 79 & 15 & 192 & ? & 172 & \\
\hline 17a. 0.0 (2) & 00 & 2 & reaction & 77.5 & 1.0 & 190 & 2 & 169 & 0 \\
\hline$M . M$ (1) & 60 & $\mathbf{M}$ & Diahetes & 109 & 5 & & & 248 & 3 \\
\hline & & & mellitus & 104 & & & & 245 & 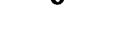 \\
\hline$M \quad R$ (2) & 27 & $F$ & & 219 & 2 & 281 & 2 & 271 & 2 \\
\hline (1) & 20 & & mellitus & 217 & & 278 & & 268 & \\
\hline $\mathrm{V}$ & 27 & $\mathbf{F}$ & (Treated) & 85 & 1 & & & 184.5 & \\
\hline 10. & 20 & 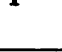 & roidism & 84 & 1 & & & 187 & 2.0 \\
\hline R I (1) & 49 & $\mathbf{F}$ & Neuro- & 73 & 3 & & & 161 & -4 \\
\hline N. L. (1) & $\Rightarrow$ & $F$ & dermatitis & 70 & & & & 165 & \\
\hline V.S. & 43 & $\mathbf{F}$ & Depressive & 80 & -1 & 188.5 & -0 & 176 & -55 \\
\hline v.s. & 40 & & reaction & 81 & -1 & 197.5 & & 181.5 & -3.0 \\
\hline M. M. (2) & 60 & $\mathbf{M}$ & Diabetes & - & . & & & 236 & -10 \\
\hline & & & mellitus & (117) & & & & 246 & \\
\hline Ma B (1) & 55 & $\mathbf{F}$ & Paranoid & 78 & 1 & 207 & -10 & 189 & -105 \\
\hline Mina. $0 .(1)$ & J & & +Cortisone & 77 & 1 & 217 & -10 & 199.5 & -10.0 \\
\hline Mean & Stan & $\operatorname{ard} I$ & viatinn & $100.7 \pm 43.4$ & $30+34$ & $206.4 \pm 36.4$ & $11+01$ & $197.0 \pm 37.7$ & \\
\hline tretain a & Neant & and 2 & Valtivir & $97.7 \pm 42.5$ & $0.0 \pm 0.4$ & $\overline{205.3 \pm 39.0}$ & $1.1 \pm 9.1$ & $\overline{194.0 \pm 39.5}$ & \\
\hline
\end{tabular}


ard error of the mean of 30 consecutive duplicate determinations was $1.48 \mathrm{mg}$. per cent for values below 120 per cent, and $1.76 \mathrm{mg}$. per cent for values above $120 \mathrm{mg}$. per cent. In one-third of the experiments the determination of known amounts of glucose added to whole blood was performed with an average recovery of 94 per cent.

\section{RESULTS}

On 19 of 40 occasions intravenous administration of $50 \mathrm{ml}$. of a 50 per cent solution of glucose was followed within 5 minutes by cessation of gastric hunger contractions. This "contractions inhibited" group is listed in Table I together with age, sex, diagnosis and glucose values of its members. In 14 of these 19 experiments there was a return of contractions $47 \pm 12$ minutes after their inhibition. In no case had previous rapid intravenous injection of $70 \mathrm{ml}$. of isotonic saline, venipunctures, or finger punctures had any effect on gastric motility.

On 16 occasions gastric hunger contractions persisted for at least 20 minutes following glucose administration. The data for the "contractions persist" group are listed in Table II. Five experiments in which contractions ceased between 5 and 20 minutes after glucose injection were considered inconclusive and are not included in either group.

\section{Fasting capillary-venous glucose differences}

A measure of the reproducibility of the capillaryvenous glucose differences is obtained from the fasting values noted in Tables I and II. Thus,

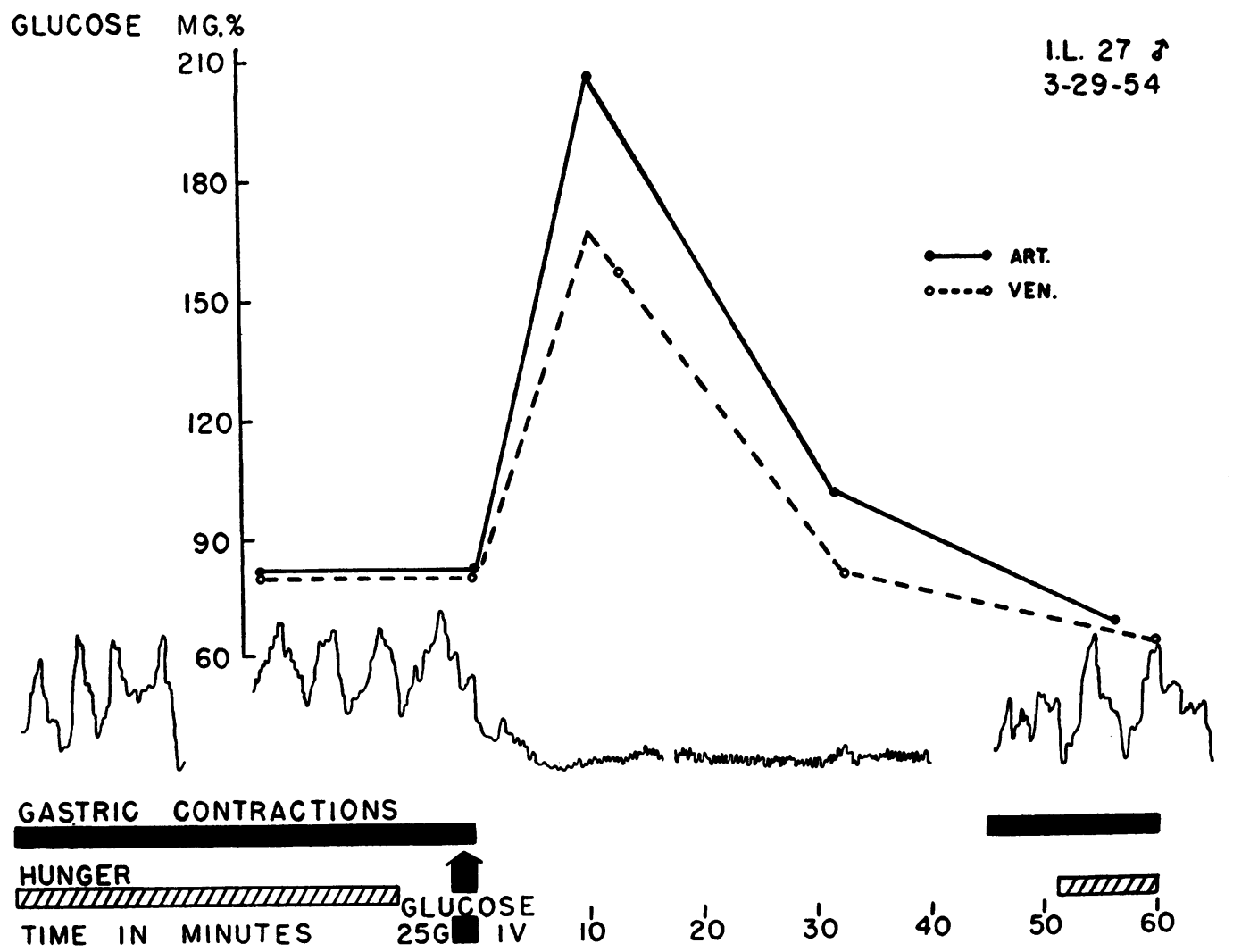

Fig. 1. (Subject I. L.) Cessation of Gastric Hunger Contractions Following the Intravenous Administration of 50 ML. OF a 50 PER CENT Solution of Glucose

Ten minutes after the injection the rise in blood glucose is associated with a capillary-venous difference of $34 \mathrm{mg}$. per cent. When blood glucose levels fall, this difference decreases rapidly, reaching a value of $8 \mathrm{mg}$. per cent at the time gastric hunger contractions return, 45 minutes after injection.

The sample tracings are from a kymograph revolving at a speed of one inch per minute. The black bar represents the actual time relations of the contractions to blood glucose levels and to the subjective experience of hunger which is indicated by the cross-hatched bar. 


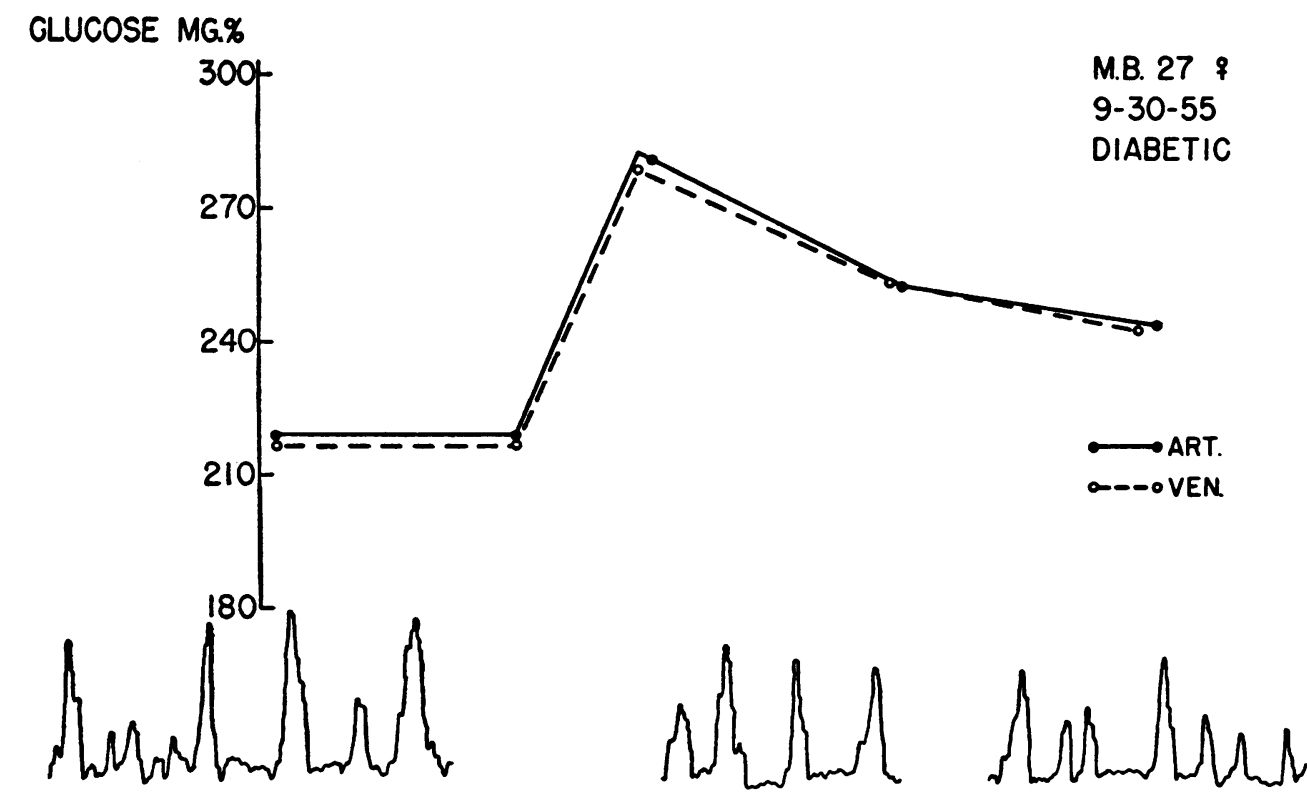

\section{GASTRIC CONTRACTIONS}

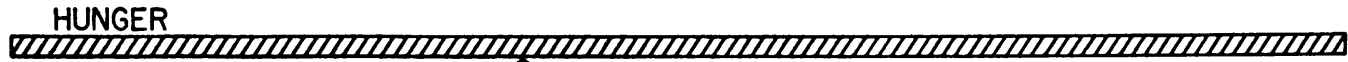

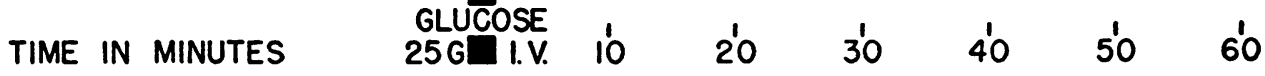

Fig. 2. (Subject M. B.) Persistence of Gastric Hunger Contractions in a Diabetic Subject Following the Intravenous Administration of 50 mL. of a 50 Per cent Solution of GLucose

Although blood glucose levels rise considerably over the fasting hyperglycemia, the capillaryvenous difference remains small.

although capillary and venous values are somewhat higher in the "contractions persist" group because of its higher percentage of diabetic subjects, the capillary-venous differences of the two groups do not differ. Their small value $(3.0 \pm$ $3.0 \mathrm{mg}$. per cent ${ }^{2}$ for the "contractions inhibited" group and $3.0 \pm 3.4 \mathrm{mg}$. per cent for the "contractions persist" group) indicates negligible peripheral removal of glucose in the fasting state, and corresponds closely to the findings of other investigators $(9-11,15)$.

Early capillary-venous glucose differences (10 and 20 minutes following glucose injection)

Higher capillary-venous glucose differences occur in the "contractions inhibited" group than in the "contractions persist" group at both 10 and 20

\footnotetext{
2 Standard deviation.
}

minutes even though there is considerable overlap. The means of the capillary-venous glucose differences of the two groups at 10 minutes are $11.6 \pm$ $10.2 \mathrm{mg}$. per cent for the "contractions inhibited" experiments, and $1.1 \pm 9.1 \mathrm{mg}$. per cent for the "contractions persist" experiments. At 20 minutes the mean capillary-venous difference of the "contractions inhibited" group is $10.5 \pm 8.3 \mathrm{mg}$. per cent, while that of the "contractions persist" group is $3.1 \pm 8.2 \mathrm{mg}$. per cent. Differences between the two groups are significant at the 5 per cent level. ${ }^{3}$ Graphic illustration of these findings is provided by Figures 1 and 2 .

${ }^{3}$ In each comparison in this report capillary-venous glucose differences ranked in order of their magnitude were subjected to statistical analysis by means of the rank " $t$ " test. This instrument was considered most appropriate for the material since it requires no assumption of a normal distribution (16). 
A comparison between capillary-venous glucose differences of subjects with hunger contractions and those without them was made at two other times. The results provide a further test of the glucostatic theory. The first comparison, within the "contractions inhibited" group, was of early capillary-venous glucose differences (when contractions were absent) and such differences at the time contractions returned. The second comparison, also within the "contractions inhibited" group, was of capillary-venous differences when contractions returned, and when they did not.

Comparison of early (10-and 20-minute) capillaryvenous glucose differences with those occurring later

In 14 of the experiments in which contractions were inhibited there was a return of gastric motility an average of 47 minutes after glucose injection. Capillary and venous glucose levels were interpolated for the time at which contractions returned in each subject, and these "contractions return" values are recorded in Table I. Comparison of capillary-venous differences at 10 and 20 minutes with those at the time contractions returned reveals that the latter values are usually lower. The means are $10.0 \pm 12.5 \mathrm{mg}$. per cent at 10 minutes and $9.6 \pm 10.0 \mathrm{mg}$. per cent at 20 minutes. The means of the "contractions return" group are $1.4 \pm 4.1 \mathrm{mg}$. per cent for the 7 subjects whose 10-minute values are available, and $3.6 \pm$ $5.2 \mathrm{mg}$. per cent for the group as a whole. The difference between groups is not significant at the 5 per cent level $(P=0.11$ and 0.12$)$.

\section{Comparison of capillary-venous glucose differ- ences when contractions returned and when they did not}

In 5 of the 19 experiments in which contractions were inhibited they did not return during the period of observation. Table I lists the capillaryvenous glucose differences 47 minutes after glucose injection for these 5 experiments. These values are considerably larger than the capillaryvenous differences at the time contractions returned (an average of 47 minutes after injection) in the other 14 experiments of the "contractions inhibited" group. The mean of the capillary-venous differences for the group in which contractions did not return is $12.2 \pm 3.6 \mathrm{mg}$. per cent, as compared with $3.6 \pm 5.2 \mathrm{mg}$. per cent for the subjects in whom contractions did return. The difference between groups is significant at the one per cent level.

\section{DISCUSSION}

\section{Methodology}

The methods used in this investigation represent an attempt to devise a standardized procedure for the study of those problems of appetite and hunger which can be approached through the measurement of gastric hunger contractions. This procedure involves the application of the stimulus of intravenous glucose administration to human subjects, and the measurement of two variables, (a) peripheral capillary-venous glucose differences, and (b) gastric hunger contractions. The significance and reliability of the stimulus as well as of the two indices has been explored.

Studies of carbohydrate metabolism have long utilized peripheral arteriovenous glucose differences, ar their equivalent, capillary-venous differences $(9,11,17-19)$. In the presence of a constant blood flow, ${ }^{4}$ such differences depend upon the rate at which glucose is removed by the tissues, and these removal rates are closely related to the general level of carbohydrate stores. Thus, during fasting capillary-venous glucose differences are very small, and they increase in magnitude with carbohydrate repletion, notably during a glucose tolerance test, or following a meal. The relevance of these findings to the present study has been established by Van Itallie, Beaudoin, and Mayer who demonstrated an excellent temporal correlation between such variations in capillaryvenous glucose differences and naturally occurring states of hunger and satiety (21).

Recording of gastric hunger contractions is a convenient and objective measure of the hunger state. It has, however, certain limitations. In the

4 The assumption of a constant blood flow under the conditions of this investigation is supported by a recent study which showed that the intravenous injection of enough 40 per cent glucose solution to raise the blood sugar to $300 \mathrm{mg}$. per cent (higher than that recorded here) had no effect on the skin temperature of the fingers, hands or forearms (20). It seems reasonable that the far more stable blood flow of the muscles of this area would not be affected. 
first place, the contractions, like the subjective experience of hunger, are not constantly present even in the fasting state, but occur periodically on the average of one-half out of every two hours. Furthermore, they are subject to inhibition by a variety of stimuli, most prominently unpleasant sensations and emotions. It is thus possible that, following the injection of any agent, nonspecific stimuli or their normal periodicity will result in a cessation of hunger contractions, and this could be erroneously attributed to the agent. The injection procedure previously described was used in an effort to minimize such inhibition of contractions. A further effort was made to minimize the importance of nonspecific stimuli by including in the "contractions inhibited" group only those instances in which contractions ceased within 5 minutes, and by confining the "contractions persist" group to those subjects whose contractions lasted longer than 20 minutes.

The administration of glucose by the intravenous route adds further difficulties of method. This technique provides a rapid and predictable method of raising blood glucose levels, but the hyperglycemia so obtained may not correspond to that following the ingestion of food or even of glucose. In the first place the injected glucose is strongly hypertonic and in this respect dissimilar to that obtained by intestinal absorption. This very hypertonicity may have inhibitory effects on gastric hunger contractions. In the second place, the glucose enters the body through a peripheral route, differing from its absorption through the intestinal wall following oral administration. Thirdly, the rapid rise in blood glucose levels is distinctly unphysiological. Finally, the glucose so administered may be metabolized in a different manner from that present in the blood under normal circumstances. Scow and Cornfield, for example, have demonstrated that orally administered glucose is removed from the blood three times as rapidly as glucose injected intravenously (22). The small size of the capillary-venous differences reported here is additional evidence for slower removal following intravenous injection of glucose; they are far smaller than those reported following oral administration (11)..$^{5}$

\footnotetext{
- An alternative explanation of the small capillaryvenous differences is suggested by the observation that, following intravenous injection of a non-metabolizable
}

Furthermore, this occurs contrary to predictions based on the law of mass action, for the levels following injection are far higher than those following oral administration.

\section{Significance}

A relationship between gastric hunger contractions, hunger and carbohydrate metabolism was first postulated more than 30 years ago. Certain observations from clinical medicine support this view; depletion of available carbohydrate offers an explanation of hunger in such diverse conditions as starvation, unregulated diabetes mellitus, cortisone therapy and insulin hypoglycemia. The extensive experimental data relating to this problem have been reviewed by Mayer $(7,24)$ and by Grossman (25).

Certain observations of earlier workers indicated that it might be fruitful to investigate further the relation between glucose and gastric hunger contractions. As has been noted, Bulatao and Carlson reported that intravenous administration of glucose abolished hunger contractions in normal, but not in pancreatectomized, dogs. Independent confirmation of this finding was reported (4), but later, careful work has cast doubt upon its validity $(5,6)$. On one matter there has been unanimity: all reports have agreed that glucose abolishes gastric hunger contractions which have been induced by insulin hypoglycemia (4-6, $26,27)$. Since insulin administration presumably increases the rate at which glucose is utilized (18, $28)$, this observation suggests that it is the utilization rate which determines the effect of intravenous glucose on gastric motility. None of these studies, however, reported an attempt to measure such utilization.

The current study is apparently the first attempt to combine observation of the effects of glucose injection on gastric motility with an index of glucose utilization. This has made it possible to correlate capillary-venous glucose differences and gastric hunger contractions in three different con-

sugar, diffusion from the extravascular compartment back into the blood stream results in negative capillary-venous differences (23). Some such diffusion phenomenon may occur even following glucose administration to normal subjects, and seems the most reasonable explanation of the negative capillary-venous differences observed in the diabetic subjects. 
texts. Although none of these correlations is conclusive, and one is not even statistically significant, each points to a tendency for gastric quiescence to be associated with higher capillary-venous glucose differences than are found in subjects in whom hunger contractions persisted.

If the phenomena of hunger and satiety are distinguished as current theory demands (29), these findings are relevant chiefly to the latter function. They suggest that metabolic events accompanying increased peripheral removal of glucose may play a role in the normal satiety mechanism. They do not support the view that depletion of carbohydrate stores of the order produced by an overnight fast is the sole, or even a sufficient, stimulus for hunger. Fasting subjects (with negligible capillary-venous glucose differences) spent many hours during the control periods with neither the subjective experience of hunger nor the presence of gastric hunger contractions. This does not contradict the position that carbohydrate depletion is a necessary precondition of hunger. The available evidence would support this view.

The study provides evidence that a fall in blood glucose, even at hyperglycemic levels, may induce hunger contractions. Although there is general agreement that hypoglycemia induced by insulin is associated with gastric hunger contractions, it has only once been suggested that glucose administration itself can be a stimulus to such contractions, and this only during the hypoglycemic phase (5). However, in the group with a rapid shrinking of the capillary-venous glucose differences, the return of contractions an average of 47 minutes after their abolition is sooner than would be predicted from the previous spontaneous gastric activity of these subjects.

The low order of statistical significance attached to the findings of this study despite a relatively large number of observations may indicate that metabolic events accompanying increased peripheral capillary-venous glucose differences play an unimportant role in the inhibition of gastric hunger contractions. On the other hand, limitations of method could give the same result. Foremost among these limitations has been the inability to reproduce the pattern of glycemia, including the large capillary-venous glucose differences, which normally follows the ingestion of food. . If the present findings are correctly interpreted, an agent capable of such effects should abolish gastric hunger contractions even at lower glucose levels than those which follow its intravenous administration. It has recently been shown that glucagon has this effect upon blood glucose (30), and preliminary study has indicated that its administration predictably abolishes gastric hunger contractions (31). Details of these findings will be the subject of a further report.

\section{SUMMARY AND CONCLUSIONS}

1. Intravenous administration of $50 \mathrm{ml}$. of a 50 per cent solution of glucose was followed within 5 minutes by the cessation of gastric hunger contractions in 19 of 40 experiments on a group of diabetic and non-diabetic human subjects.

2. At 10 and 20 minutes following injection, the peripheral capillary-venous glucose differences in the group of 19 subjects in which contractions were inhibited were significantly higher than those of the group in which contractions persisted.

3. In 14 of the 19 experiments in which contractions were inhibited, they returned at an average of 47 minutes after injection. Capillaryvenous glucose differences at 10 and 20 minutes after injection were usually higher than those at the time contractions returned, but this difference was not significant at the 5 per cent level.

4. Capillary-venous glucose differences in the 5 experiments in which contractions remained inhibited were significantly higher than those in the 14 experiments in which they returned.

5. These findings support the view that metabolic events accompanying increased peripheral removal of glucose have an inhibiting effect on gastric hunger contractions. Events associated with decreased removal, on the other hand, are not a sufficient stimulus for the production of gastric hunger contractions, although they appear to be a necessary precondition.

\section{REFERENCES}

1. Cannon, W. B., and Washburn, A. L., An explanation of hunger. Am. J. Physiol., 1912, 29, 441.

2. Carlson, A. J., The control of hunger in health and disease. Chicago, Univ. Chicago Press, 1916.

3. Bulatao, E., and Carlson, A. J., Contributions to the physiology of the stomach. Influence of experimental changes in blood sugar level on gastric 
hunger contractions. Am. J. Physiol., 1924, 69, 107.

4. Templeton, R. D., and Quigley, J. P., The action of insulin on the motility of the gastrointestinal tract. II. Action on the Heidenhain pouch. Am. J. Physiol., 1930, 91, 467.

5. Quigley, J. P., and Hallaran, W. R., The independence of spontaneous gastro-intestinal motility and blood sugar levels. Am. J. Physiol., 1932, 100, 102.

6. Mulinos, M. G., The gastric hunger mechanism. IV. The influence of experimental alterations in blood sugar concentration on the gastric hunger contractions. Am. J. Physiol., 1933, 104, 371.

7. Mayer, J., Genetic, traumatic and environmental factors in the etiology of obesity. Physiol. Rev., 1953, 33, 472.

8. Mayer, J., Glucostatic mechanism of regulation of food intake. New England J. Med., 1953, 249, 13.

9. Foster, G. L., Studies on carbohydrate metabolism. I. Some comparisons of blood sugar concentration in venous blood and in finger blood. J. Biol. Chem., 1923, 55, 291.

10. Langner, P. H., Jr., and Fies, H. L., Blood sugar values of blood obtained simultaneously from the radial artery, antecubital vein, and the finger. Am. J. Clin. Path., 1942, 12, 559.

11. Somogyi, M., Studies of arteriovenous differences in blood sugar. I. Effect of alimentary hyperglycemia on the rate of extrahepatic glucose assimilation. J. Biol. Chem., 1948, 174, 189.

12. Somogyi, M., Determination of blood sugar. J. Biol. Chem., 1945, 160, 69.

13. Somogyi, M., A new reagent for the determination of sugars. J. Biol. Chem., 1945, 160, 61.

14. Nelson, N., A photometric adaptation of the Somogyi method for the determination of glucose. J. Biol. Chem., 1944, 153, 375.

15. Bell, D. M., Femoral arteriovenous sugar differences in fasting human beings. J. Lab. \& Clin. Med., 1952, 40, 337.

16. Bross, I. D. J., Is there an increased risk? Federation Proc., 1954, 13, 815.

17. Bang, I., Der Blutzucker, 1913, Wiesbaden, Bergmann.

18. Bell, D. M., and Burns, T., Effect on femoral a-v glucose difference of insulin injected into an ante- cubital vein and into a femoral artery. J. Clin. Invest., 1952, 31, 717.

19. Himsworth, H. P., The mechanism of diabetes mellitus. Lancet, 1939, 2, 1, 65, 118, and 171.

20. Törnblom, N., and Hellman, S., On the mechanism of diabetes in obesity. Studies on arteriovenous glucose difference in normals and in obese diabetics. Acta med. Scandinav., 1953, 147, 331.

21. Van Itallie, T. B., Beaudoin, R., and Mayer, J., Arteriovenous glucose differences, metabolic hypoglycemia and food intake in man. Am. J. Clin. Nutrition, 1953, 1, 208.

22. Scow, R. O., and Cornfield, J., Quantitative relations between the oral and intravenous glucose tolerance curves. Am. J. Physiol., 1954, 179, 435.

23. Van Itallie, T. B., Morgan, M. C., and Dotti, L. B., Peripheral diffusion characteristics of sucrose in man. Federation Proc., 1954, 13, 156.

24. Mayer, J., Regulation of energy intake and the body weight: The glucostatic theory and the lipostatic hypothesis. Ann. New York Acad. Sc., 1955, 63, 15.

25. Grossman, M. I., Integration of current views on the regulation of hunger and appetite. Ann. New York Acad. Sc., 1955, 63, 76.

26. Quigley, J. P., and Templeton, R. D., Action of insulin on the motility of the gastrointestinal tract. III. a. Action on the pyloric pouch. b. Action on the stomach following double splanchnicotomy. Am. J. Physiol., 1930, 91, 475.

27. Regan, J. F., The action of insulin on the motility of the empty stomach. Am. J. Physiol., 1933, 104, 90.

28. Stadie, W. C., and Zapp, J. A., Jr., The effect of insulin upon the synthesis of glycogen by rat diaphragm in vitro. J. Biol. Chem., 1947, 170, 55.

29. Brobeck, J. R., Neural regulation of food intake. Ann. New York Acad. Sc., 1955, 63, 44.

30. Van Itallie, T. B., Morgan, M. C., and Dotti, L. B., Effect of glucagon on peripheral utilization of glucose in man. J. Clin. Endocrinol. \& Metab., $1955,15,28$.

31. Stunkard, A. J., Van Itallie, T. B., and Reis, B. B., The mechanism of satiety: Effect of glucagon on gastric hunger contractions in man. Proc. Soc. Exper. Biol. \& Med., 1955, 89, 258. 